\title{
CT-Guided Percutaneous Fine-Needle Aspiration Biopsy of the Inferior Vena Cava Wall: A Posterior Coaxial Approach
}

\author{
Sebastian Kos · Deniz Bilecen · Daniel Baumhoer • \\ Nicolas Guillaume · Augustinus L. Jacob
}

Received: 14 November 2008/ Accepted: 14 January 2009/Published online: 12 February 2009

(C) Springer Science+Business Media, LLC 2009

\begin{abstract}
A 72-year-old man was referred to our department with an incidentally diagnosed bronchogenic carcinoma of the right upper lobe. Positron emission tomography (PET) combined with computed tomography (PET-CT) revealed an unexpected hot spot in the ventral wall of the infrarenal segment of the inferior vena cava (IVC). Diagnostic biopsy of this lesion was performed under CT guidance with semiautomated $20 \mathrm{G}$ fine-needle aspiration (FNA) through a 19G coaxial needle. Cytology revealed few carcinoma cells, which led to the remarkable diagnosis of a distant metastasis to the IVC wall. Both the immediate postinterventional CT control and the further surveillance period of the patient were unremarkable; in particular, no signs of bleeding complications were detected. We conclude that coaxial FNA of an IVC wall lesion is technically feasible and may even help diagnose distant metastasis.
\end{abstract}

Keywords Inferior vena cava $\cdot$ Metastasis ·

Coaxial FNA · CT guided

\section{Introduction}

Primary tumors of the inferior vena cava (IVC) are rare and generally comprise leiomyosarcomas and leiomyomas of

S. Kos $(\bowtie) \cdot$ D. Bilecen · A. L. Jacob

Institute of Radiology, University Hospital Basel,

Petersgraben 4, 4031 Basel, Switzerland

e-mail: skos@gmx.de

\section{Baumhoer}

Institute of Pathology, University Hospital Basel,

Schönbeinstrasse 40, 4003 Basel, Switzerland

N. Guillaume

Institute of Nuclear Medicine, University Hospital Basel,

Petersgraben 4, 4031 Basel, Switzerland the vessel wall $[1,2]$. Whereas tumor thrombi caused by continuos spread of cancer cells are common events in several malignant tumors like renal cell carcinoma and hepatocellular carcinoma, metastases to the vascular wall have only rarely been described [1, 3, 4].

For the histological diagnosis of such a lesion, which is usually detected incidentally during staging procedures, different approaches were described. Generally, the diagnosis is made on the surgically resected specimen, but minimally invasive approaches have also been reported. These include sonogram- or computed tomography (CT)guided percutaneous fine-needle aspiration (FNA), intraluminal biopsy, and endosonographic FNA [5-7].

\section{Case Report}

A 72-year-old man was referred to our hospital with a history of systemic sclerosis. Annual conventional radiography of the thorax revealed a large lobulated mass at the hilum of the right lung, complicated by a secondary poststenotic pneumonia. Transbronchial biopsy, guided by endobronchial sonography, was performed, and a bronchogenic adenocarcinoma of the right upper lobe was subsequently diagnosed. Positron emission tomography (PET) combined with CT (PET-CT), performed for treatment planning (Fig. 1A-C), showed a high fluorodeoxyglucose (FDG) uptake of the bronchogenic carcinoma and additionally of an ovoid lesion in the infrarenal segment of the IVC. Further characterization of the second lesion was achieved by magnetic resonance tomography, which demonstrated a $0.6 \times 0.9-\mathrm{cm}$ hypointense thickening in the ventral venous wall (Fig. 1D). Because the differential diagnosis comprised a primary malignant tumor of the vein or a distant metastasis to the venous wall by the bronchogenic carcinoma, biopsy had to be performed. 
Fig. 1 PET-CT (A-C) and MR (D) imaging. Coronal view (A, C) and axial view $(\mathbf{B}, \mathbf{D})$. PETCT documented the high FDG uptake of the primary bronchogenic carcinoma of the right upper lobe (arrowhead in A). It also showed a second FDG-positive, round lesion in the IVC (arrows in A-C). Native magnetic resonance imaging that used a TrueFISP sequence showed that the lesion is hypointense and lies within the ventral wall of the vein, thereby having a convex appearance and forming an obtuse shoulder with the hyperintense lumen of the vein (arrow in D)
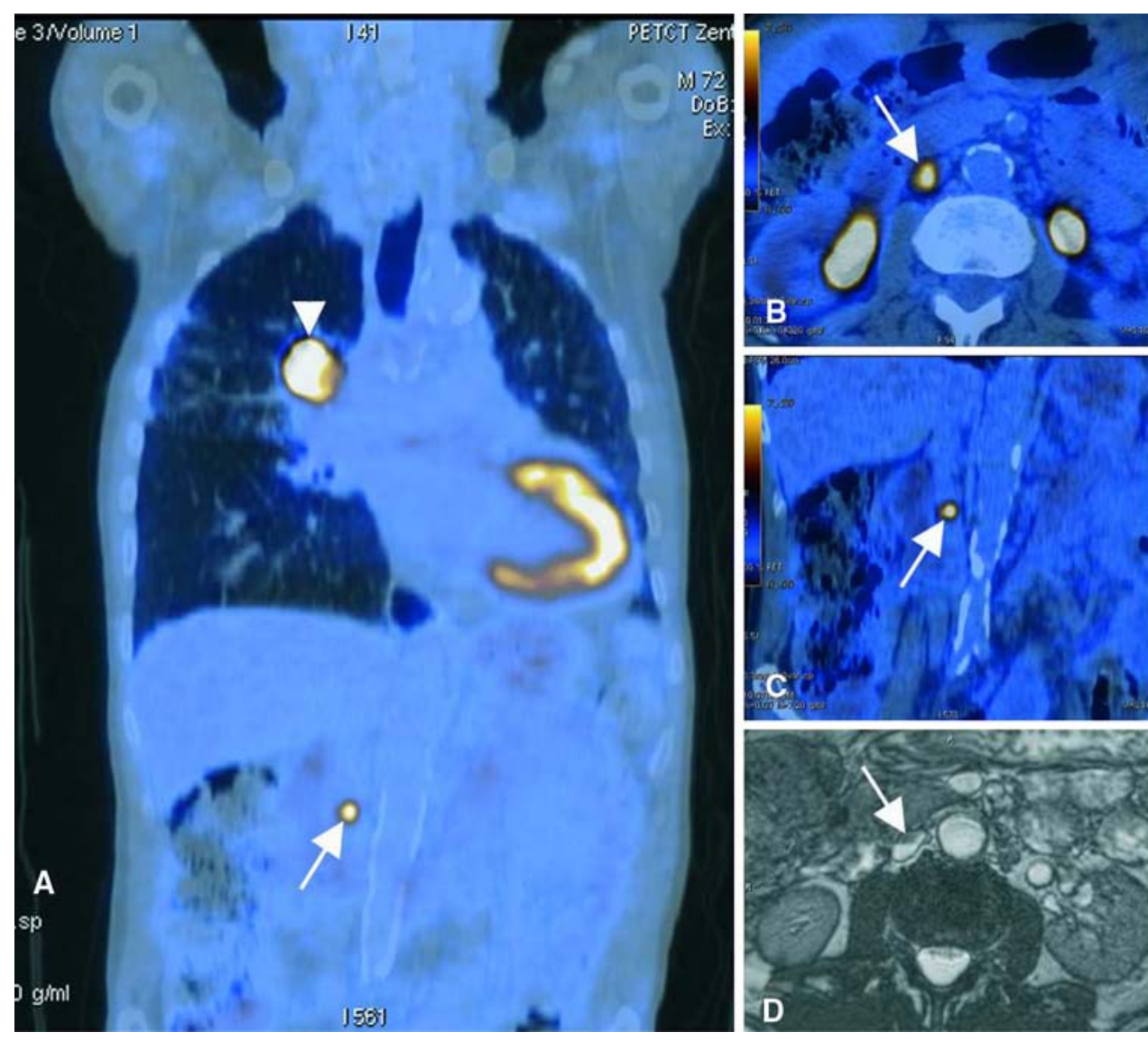

Percutaneous CT-guided intervention was performed in a 64-slice scanner (Somatom Sensation 64, Siemens Medical Solutions, Germany). The patient was positioned prone with arms fixed above his head. Conscious sedation (midazolam/ Dormicum and alfentanil/Rapifen) was administered intravenously, and vital parameters (heart rate, blood pressure, peripheral oxygenation) were continuously monitored.

For procedure planning, intravenous contrast-enhanced $\mathrm{CT}$ of the upper abdomen was performed in both the arterial (after $15 \mathrm{~s}$ ) and the portal-venous phase (after $65 \mathrm{~s}$ ). The lesion in the venous wall was only poorly depicted in the portal-venous phase, which was then used for further planning. By means of an X-ray-absorbing grid on the skin left to the spine, the cutaneous access point was chosen and a puncture trajectory planned. The cutis was locally anesthetized with $9 \mathrm{ml}$ of mepivacaine $1.5 \%$ diluted with $1 \mathrm{ml}$ of natrium bicarbonate $8.4 \%$. Along the prior planned trajectory and according to its lengths, a 19G 11.4$\mathrm{cm}$ coaxial introducer needle (Medical Device Technologies Inc., Gainesville, FL) was chosen and advanced through a posterior paravertebral access (Fig. 2). This access was chosen because it offered a short length and had the least vulnerable structures in the access path compared with other approaches (e.g., anterior or lateral). Intermittent low-dose CT controls (slice thickness $3 \mathrm{~mm}$ ) documented the exact advancement of the needle to the dorsal wall of the IVC, avoiding adjacent nerve roots, the renal vessels, and ureter. The dorsal wall of the vena cava was then punctured and the tip of the coaxial needle positioned in the middle third of the caval lumen, pointing toward the target lesion. After removal of the inner stylet, a 20G 15$\mathrm{cm}$ Menghini-type semiautomatic biopsy device (Biomol, H.S. Hospital Service, Rome, Italy) was inserted for biopsy. To reduce the risk of air embolism through the coaxial needle, its introducer hub was plugged with a finger immediately after stylet removal until the biopsy needle was inserted. Three biopsy specimens were taken from different portions of the lesion by a slight movement of the coaxial needle tip in a left-right and cranio-caudal direction. CT controls were performed to document the correct position of the needle. Because no solid specimens were obtained, the needle was flushed each time with a cytological fixating solution and sent for cytological analysis.

The procedure lasted $51 \mathrm{~min}$ from the initial CT planning to the postinterventional control. This follow-up CT showed no signs of complications. In particular, no bleeding was evident along the puncture trajectory adjacent to the IVC.

The patient tolerated the procedure well and was monitored for a further $6 \mathrm{~h}$ after the procedure, without evidence of complications. Cytological analysis showed 

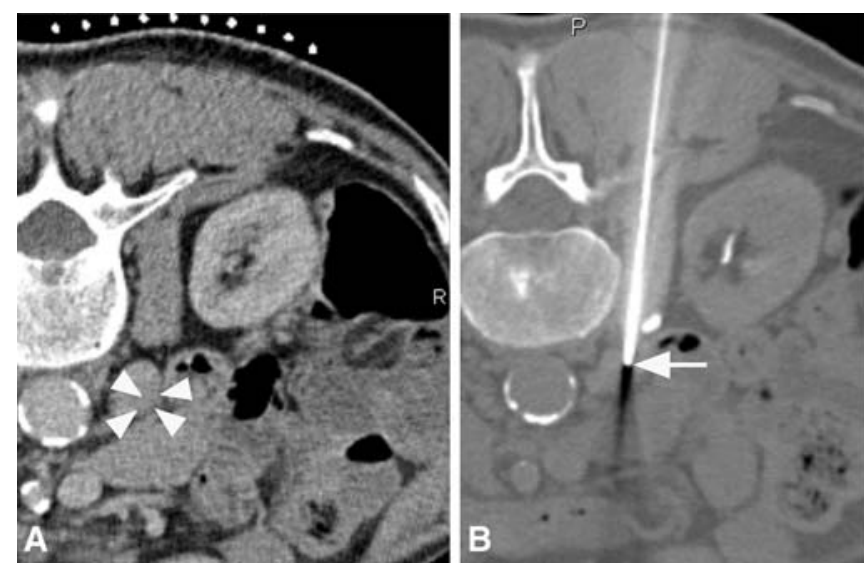

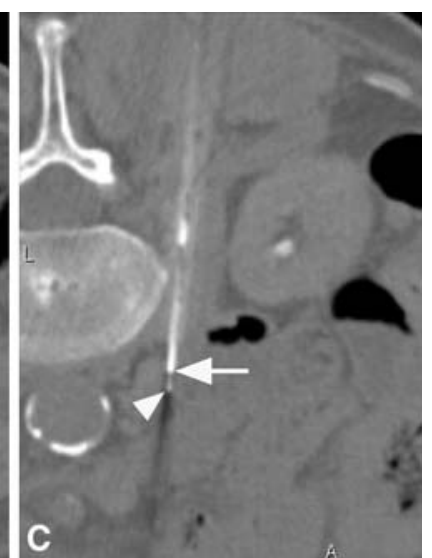

Fig. 2 CT-guided percutaneous biopsy performed using a coaxial technique (A-C, axial views). Initial contrast-enhanced CT in a portal-venous phase only poorly demarcates the hypodense lesion in the ventral wall of the IVC (arrowheads in A). In a first step, a 19G 11.4-cm coaxial needle is advanced through the dorsal wall of the

few carcinoma cells with immunocytochemical expression of thyroid transcription factor 1 (TTF1), a typical marker protein for adenocarcinomas of the lung.

\section{Discussion}

The cytological and histological assessment of tumors and its metastatic lesions is mandatory to allow tumor staging and therapy planning. Regarding tumors of the vena cava, different biopsy approaches have been described. The diagnosis is most often made from specimens obtained during surgical resection, but in addition, minimally invasive methods such as intraluminal biopsy and endosonographic FNA have been described to be promising alternatives [5-7]. Al-Rikabi et al. [6] performed a CT-guided FNA in a case of leiomyosarcoma of the IVC, but did not describe the exact technique applied. To our knowledge, this is the first report of obtaining a CT-guided FNA biopsy sample of a metastatic tumor to the vascular wall.

Because the venous wall is punctured only once and tissue aspiration is then performed through the coaxial needle already in place, the coaxial technique seems feasible and safe. Further, biopsy samples can be taken from various positions in the targeted lesion without repetitive damage to the vascular wall. By choosing a percutaneous CT-guided approach, standard equipment can be used, and additional costs (e.g., for endosonographic devices) can be avoided. We did not use a large-bore true-cut biopsy system in this case because of an increased risk of bleeding. In addition, because the duodenum is located directly adjacent to the ventral cava wall, a true-cut biopsy may increase the risk to cause intestinal perforation, or may even create a cavointestinal fistula. vein and into the lumen (arrow in B). In the second step, the biopsy is performed with a 20G 15-cm Menghini-type needle (arrowhead in C) through the coaxial needle (arrow in C). Biopsy via the coaxial needle can be safely repeated because repetitive perforation of the venous wall is not necessary

In general, FNA biopsies performed under CT guidance are known to be precisely steerable and therefore carry a low risk of injuring structures (e.g., nerves, kidney, ureter, renal vessels) adjacent to the planned trajectory [8]. Risk is further reduced by using a coaxial approach, making only a single needle passage into the IVC necessary. By puncturing the dorsal wall of the IVC, bleeding complications may occur. Those are theoretically minimized by only perforating the venous wall once. Relevant bleeding complications have not been described in association with this technique; such complications are unlikely because in the past, even aortographies have been routinely performed through direct accesses to the abdominal aorta [9]. By establishing a direct access to the low-pressure venous system, air embolism may occur as a complication [10]. This risk was minimized by keeping the venous access constantly plugged by either the inner stylet of the coaxial needle, the index finger of the operator, or the biopsy device, a technique being routinely performed for percutaneous lung biopsies. In the future, a modified coaxial needle with an intrinsic sealing mechanism would facilitate this procedure.

In the present case, the cava wall lesion revealed few carcinoma cells positive for TTF1, which is a highly specific marker for adenocarcinomas of the lung [11, 12]. The lesion therefore represents a distant metastasis (cT4 cN0 pM1) of the bronchogenic carcinoma, an entity that has not been described before. By using FDG PET-CT for tumor staging, the asymptomatic metastasis was detected early.

In conclusion, we demonstrate that even lesions in the ventral wall of the vena cava may be safely reached via a percutaneous CT-guided technique, and a cytological diagnosis can be achieved performing repetitive FNA biopsy through a coaxial access. 


\section{Reference}

1. Burke AP, Virmani R (1995) Neoplasms of large arteries and veins, and tumor angiogenesis. In: Stehbens WE, Lie JT (eds) Vascular Pathology. Chapman \& Hall, Alden Press, Oxford, pp 729-738

2. Hilliard NJ, Heslin MJ, Castro CY (2005) Leiomyosarcoma of the inferior vena cava: three case reports and review of the literature. Ann Diagn Pathol 9:259-266

3. Didier D, Racle A, Etievent JP et al (1987) Tumor thrombus of the inferior vena cava secondary to malignant abdominal neoplasms: US and CT evaluation. Radiology 162:83-89

4. Cuevas C, Raske M, Bush WH et al (2006) Imaging primary and secondary tumor thrombus of the inferior vena cava: multidetector computed tomography and magnetic resonance imaging. Curr Probl Diagn Radiol 35:90-101

5. Shimoda H, Oka K, Otani S et al (1998) Vascular leiomyosarcoma arising from the inferior vena cava diagnosed by intraluminal biopsy. Virchows Arch 433:97-100

6. Al-Rikabi A, Hussain AA, Buchler M et al (2007) Primary leiomyosarcoma of the inferior vena cava: report of a case diagnosed by fine needle aspiration cytology and confirmed by histopathologic examination. Acta Cytol 51:477-479
7. Jenssen C, Siebert C, Bartho S (2008) Leiomyosarcoma of the inferior vena cava. Diagnosis using endoscopic ultrasound-guided fine-needle aspiration biopsy. Dtsch Med Wochenschr 133: 769-772

8. Sheikh M, Sawhney S, Dey P et al (2000) Deep-seated thoracic and abdominal masses: usefulness of ultrasound and computed tomography guidance in fine needle aspiration cytology diagnosis. Australas Radiol 44:155-160

9. Viville C, Gillet M, Reins R (1966) Complications of lumbar aortography (or direct aortography) and their prevention. J Radiol Electrol Med Nucl 47:289-308

10. Kau T, Rabitsch E, Celedin S et al (2008) When coughing can cause stroke-a case-based update on cerebral air embolism complicating biopsy of the lung. Cardiovasc Intervent Radiol 31:848-853

11. Reis-Filho JS, Carrilho C, Valenti C et al (2000) Is TTF1 a good immunohistochemical marker to distinguish primary from metastatic lung adenocarcinomas? Pathol Res Pract 196:835-840

12. Perner S, Wagner P, Soltermann A et al (2009) TTF1 expression in non-small cell lung carcinoma: association with TTF1 gene amplification and improved survival. J Pathol 217:65-72 